\title{
Ultrasonic Positioning System Implementation and Dynamic 3D Visualization
}

\author{
Andon Lazarov ${ }^{1,2}$, Dimitar Minchev ${ }^{1}$, Atanas Dimitrov ${ }^{1}$ \\ ${ }^{1}$ Burgas Free University, 8000 Burgas, Bulgaria \\ ${ }^{2} \mathrm{~K}$. N. Toosi University, Tehran, Iran \\ E-mails:lazarov@bfu.bgrmitko@lbfu.bg antanas@bfu.bg
}

\begin{abstract}
The paper contributes to the design and implementation of the ultrasonic positioning system based on new multifunctional hardware components, newly released. A moving object coordinates' determination is described analytically and a matrix equation in respect to unknown coordinates with coefficients, measurement distances, is derived. Stages of data packet processing are formulated, and a pseudo pyramid of measurement distances is built. HX7TR multifunctional ultrasonic devises, transceivers, are used to implement the positioning system. A C\# program source code for coordinate determination and $3 D$ visualization is created. The algorithm for moving object coordinate computation, and its program realization as well as HX7TR ultrasonic devises can be used in development of indoor ultrasonic positioning systems embedded in IoT and robotics applications.
\end{abstract}

Keywords: Tracking system, ultrasonic positioning, matrix equations solution, moving object coordinates determination, ultrasound measurements.

\section{Introduction}

The information for location of moving and stationary objects is of particular importance in many scientific and industrial areas of human activities, robotics, storage and stock management, costumer services, remote control, medicine, etc. It stimulates active research and design of local positioning systems with varied applications. Different technologies based on propagation properties of radio, optical and acoustic waves are applied [1-4]. The accent in the present work is on acoustic waves, considering their properties such as slow propagation speed, negligible penetration in walls and low cost of the transducers. Based on their measurement capabilities as high precision (a few millimetres) and short distance, ultrasound systems are appropriate for indoor application. The distances between ultrasonic devices (transceivers), are calculated by multiplication of a signal's Time-Of-Flight (TOF) between devices with the speed of the sound. 
A summary analysis of different tracking systems, basic principles, methods, structures and tracking algorithms are suggested in [5]. Recently, algorithms for object positioning based distances' measurements have been developed, for example, Least Squares (LS) algorithm implemented in Matlab [6], indoor localizing algorithm based on bi-phase measurements for wireless sensor networks [7]. Comparison of hybrid localization schemes using rssi, toa, and tdoa are discussed in [8].

In [9-12] there are presented an active tracking system using an ultrasonic sensing device and IEEE 802.15.4 compatible radio in wireless sensor networks, and an indoor positioning system using TOF of the ultrasonic signal to estimate the distance between a receiver node and a transmitter node.

Indoor positioning systems using ultrasound-localizing pulses demonstrate a great diversity in their topologies and applications. Design and evaluation of a robust indoor ultrasound location system and broadband ultrasonic location systems for improved indoor positioning are discussed in $[13,14]$. Multi position tracking system with ultrasonic sensor modules applying positioning technique based on transmissions from independent ultrasound beacons, and a person-tracking mobile robot using an ultrasonic positioning system are discussed in [15, 16]. A passive mobile positioning system is able to locate itself using the signatures of the received ultrasound signals and shifts in the periodicities of the signals due to the Doppler effect, to estimate its location and velocity [17].

Hybrid ultrasound and wireless system applications are of great interest for researchers. Modern cellular phones have properties of transmitting and receiving ultrasonic tones between $20-22 \mathrm{kHz}$ with minimal distortion. It means that mobile devices like smartphones and tablets passes ultrasonic positioning capabilities. An indoor ultrasonic location tracking system that can utilize off-the-shelf audio speakers to provide precise indoor position data to modern mobile devices are presented in [18-20]. In [21] capabilities of a tracking technique based on ultrasonic beacons and Android applications aimed to the personal privacy are discussed. A survey on localization for mobile wireless sensor networks is suggested in [22]. Collaborative context-aware indoor positioning techniques and indoor localization without infrastructure using the acoustic background spectrum are presented in [23-25].

In contrast to aforementioned ultrasound measurement systems, in the present work an ultrasound positioning system is analysed, implemented and dynamically visualized based on ultrasound transceivers' measurements. Matrix equations with unknowns, coordinates of moving object and coefficients, measurement distances, are derived. Stages of data packet processing are presented, and a pseudo pyramid of measured mutual distances between transceivers and distances to the moving object is constructed. HX7TR multifunctional ultrasonic transceivers are used to implement the positioning system. A C\# program source code for coordinate determination and $3 \mathrm{D}$ visualization is implemented.

The remainder of the paper is organized as follows. In Section 2, an analytical description of the moving target coordinates' determination is given. In Section 3, experimental scenario description and stages of data packet processing are 
discussed. In Section 4, a definition of the square based pyramid formed by edges and vertexes of the full oriented and weighted graph is given, C\# program implementation of 3D dynamic visualization of ultrasonic measurement system are illustrated. In Section 5, conclusion remarks are derived.

\section{Analytical description of the moving object coordinates' determination}

Consider a positioning system that consists of $n+1$ ultrasonic transceivers placed in a $3 \mathrm{D}$ coordinate system. It is assumed that one of the elements is with unknown coordinates and possibly moving, others $n$ transceivers are with preliminary defined coordinates and possibly stationary. Determination of the current coordinates of a moving object is based on $n$ distance measurements between $n$ stationary transceivers and a moving object. The distance measured from $i$ th transceiver with known coordinates to the moving object with unknown coordinates, i.e., $i$-th measurement, is defined by

$$
\hat{R}_{i}(p)=\sqrt{\left[x(p)-x_{i}(p)\right]^{2}+\left[y(p)-y_{i}(p)\right]^{2}+\left[z(p)-z_{i}(p)\right]^{2}},
$$

where $x_{i}(p), y_{i}(p)$, and $z_{i}(p)$ are the known coordinates of $i$-th transceiver, $x(p), y(p)$, and $z(p)$ are the current unknown coordinates of the moving object designated by $(n+1)$-th transceiver, $p$ denotes the moment of the measurements as well as the number of data packet containing values of $n$ measurements.

The distance measured from $j$-th transceiver with known coordinates to the moving object with unknown coordinates, i.e., $j$-th measurement, is defined by

$$
\hat{R}_{j}(p)=\sqrt{\left[x(p)-x_{j}(p)\right]^{2}+\left[y(p)-y_{j}(p)\right]^{2}+\left[z(p)-z_{j}(p)\right]^{2}},
$$

where $x_{j}(p), y_{j}(p)$, and $z_{j}(p)$ are the coordinates of $j$-th transceiver.

Square both sides of the Equation (1). The square distance from $i$-th transceiver with known coordinates to the moving object can be calculated by equation

$$
\hat{R}_{i}^{2}(p)=\left[x(p)-x_{i}(p)\right]^{2}+\left[y(p)-y_{i}(p)\right]^{2}+\left[z(p)-z_{i}(p)\right]^{2} .
$$

Similarly, square both sides of the Equation (2). The square distance from $j$-th transceiver with known coordinates to the moving object with unknown coordinates can be calculated by equation

$$
\hat{R}_{j}^{2}(p)=\left[x(p)-x_{j}(p)\right]^{2}+\left[y(p)-y_{j}(p)\right]^{2}+\left[z(p)-z_{j}(p)\right]^{2},
$$

Expressions (3) and (4) represent analytical geometrical equations of spheres with centers placed on the positions of the transceivers with known coordinates.

Based on $n$ measurements of distances, determined by $\hat{R}_{i}(p)$ and $\hat{R}_{j}(p)$, a redefined system of $n$ equations for determination of moving object's coordinates, $x(p), y(p)$, and $z(p)$ can be defined. For unambiguous determination of the moving object's coordinates, minimum three measurements by three transceivers have to be completed. The system of equations can be linearized in respect of the 
unknown coordinates by mutual subtraction of composed equations defined by (3) and (4). Subtraction of Equation (4) for $\hat{R}_{j}^{2}(p)$ from Equation (3) for $\hat{R}_{i}^{2}(p)$ yields the following generalized deferential equation:

$$
\begin{aligned}
& \hat{R}_{i}^{2}(p)-\hat{R}_{j}^{2}(p)=-2 \cdot x(p)\left[x_{i}(p)-x_{j}(p)\right]+x_{i}^{2}(p)-x_{j}^{2}(p)-2 . y(p)\left[y_{i}(p)-\right. \\
& \left.-y_{j}(p)\right]+y_{i}^{2}(p)-y_{j}^{2}(p)-2 . z(p)\left[z_{i}(p)-z_{j}(p)\right]+z_{i}^{2}(p)-z_{j}^{2}(p) .
\end{aligned}
$$

Substitute

$$
\begin{gathered}
x_{i, j}(p)=x_{i}(p)-x_{j}(p), \\
y_{i, j}(p)=y_{i}(p)-y_{j}(p), \\
z_{i, j}(p)=z_{i}(p)-z_{j}(p), \\
R_{i}^{2}(p)=x_{i}^{2}(p)+y_{i}^{2}(p)+z_{i}^{2}(p), \\
R_{j}^{2}(p)=x_{j}^{2}(p)+y_{j}^{2}(p)+z_{j}^{2}(p) .
\end{gathered}
$$

Then the Equation (5) can be rewritten as

$$
\begin{gathered}
x_{i, j}(p) \cdot x(p)+y_{i, j}(p) \cdot y(p)+z_{i, j}(p) \cdot z(p)= \\
=-\frac{1}{2}\left[\hat{R}_{i}^{2}(p)-\hat{R}_{j}^{2}(p)-R_{i}^{2}(p)+R_{j}^{2}(p)\right] .
\end{gathered}
$$

It has to be underlined that the solution of the system (8) for coordinates of moving object is unambiguous if and only if the following matrix multiplication $\left[x_{i, j}(p) \quad x_{i, j}(p) \quad x_{i, j}(p)\right]^{\mathrm{T}}\left[x_{i, j}(p) \quad x_{i, j}(p) \quad x_{i, j}(p)\right]$ is not singular, i.e.,

$$
\operatorname{det}\left\{\left[x_{i, j}(p) \quad x_{i, j}(p) \quad x_{i, j}(p)\right]^{\mathrm{T}}\left[x_{i, j}(p) \quad x_{i, j}(p) \quad x_{i, j}(p)\right]\right\}>0 .
$$

The full number of distance differences, $K$ is equal to the number of combinations of two measurements among $n$ measurements without repetition and calculated by the next combinatory expression:

$$
K=C_{n}^{k}=\frac{n !}{(n-k) ! \cdot k !},
$$

where $n \geq 3$ denotes the number of measurements (transceivers with known coordinates), $k=2$ denotes the number of measurements (transceivers) in one combination without repetition.

Indices $i$ and $j$ are used to determine combinations of two transceivers' measurements without repetition. Does not matter how to define intervals of indices $i$ and $j$ in order to determine the number of full combinations without repetition $K$, e.g., $i=[1, \ldots, n-1]$, and $j=[2, \ldots, n]$ or $i=[2, \ldots, n]$, and $j=[1, \ldots, n-1]$. A Limited number of combinations are used. For unambiguous determination of unknown coordinates the minimum number of combinations, i.e., the number of differential Equations (8) is three.

For the purposes of the experiment accomplished in the present work it is assumed that the number, $n$ of distance measurements (transceivers-moving object) that constitute the data packet is $n=4$.

If $i$ and $j$ accept values in intervals as follows $i=[2, \ldots, 4]$, and $j=[1, \ldots, 3]$, the full number of distance differences is $K=6$. Three distance differences are 
chosen to compose differential equations. Then based on Equation (8) the linear system of equations can be written -

$$
\begin{gathered}
x_{2,1}(p) \cdot x(p)+y_{2,1}(p) \cdot y(p)+z_{2,1}(p) \cdot z(p)= \\
=-\frac{1}{2}\left[\hat{R}_{2}^{2}(p)-\hat{R}_{1}^{2}(p)-R_{2}^{2}(p)+R_{1}^{2}(p)\right], \\
x_{3,1}(p) \cdot x(p)+y_{3,1}(p) \cdot y(p)+z_{3,1}(p) \cdot z(p)= \\
=-\frac{1}{2}\left[\hat{R}_{3}^{2}(p)-\hat{R}_{1}^{2}(p)-R_{3}^{2}(p)+R_{1}^{2}(p)\right], \\
x_{4,1}(p) \cdot x(p)+y_{4,1}(p) \cdot y(p)+z_{4,1}(p) \cdot z(p)= \\
=-\frac{1}{2}\left[\hat{R}_{4}^{2}(p)-\hat{R}_{1}^{2}(p)-R_{4}^{2}(p)+R_{1}^{2}(p)\right],
\end{gathered}
$$

where the following substitutions are applied:

(13) $x_{2,1}(p)=x_{2}(p)-x_{1}(p), \quad y_{2,1}(p)=y_{2}(p)-y_{1}(p), \quad z_{2,1}(p)=z_{2}(p)-z_{1}(p)$,

$$
\begin{array}{cl}
x_{3,1}(p)=x_{3}(p)-x_{1}(p), & y_{3,1}(p)=y_{3}(p)-y_{1}(p), \quad z_{3,1}(p)=z_{3}(p)-z_{1}(p), \\
x_{4,1}(p)=x_{4}(p)-x_{1}(p), & y_{4,1}(p)=y_{4}(p)-y_{1}(p), \quad z_{4,1}(p)=z_{4}(p)-z_{1}(p), \\
R_{1}^{2}(p)=x_{1}^{2}(p)+y_{1}^{2}(p)+z_{1}^{2}(p), & R_{3}^{2}(p)=x_{3}^{2}(p)+y_{3}^{2}(p)+z_{3}^{2}(p), \\
R_{2}^{2}(p)=x_{2}^{2}(p)+y_{2}^{2}(p)+z_{2}^{2}(p), \quad R_{4}^{2}(p)=x_{4}^{2}(p)+y_{4}^{2}(p)+z_{4}^{2}(p),
\end{array}
$$
form as

The system of linear Equations (10), (11), and (12) can be expressed in matrix

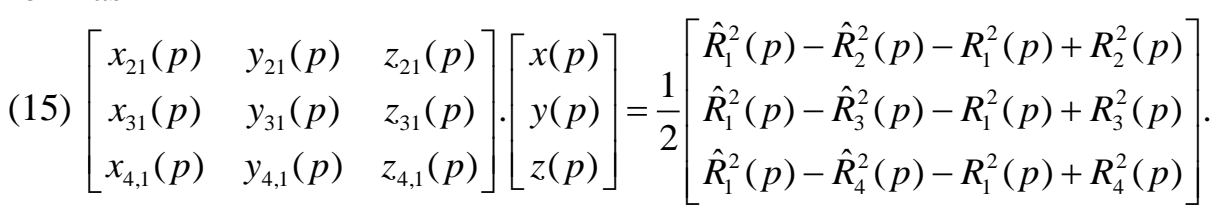

The matrix Equation (15) can be presented in a vector form as

$$
\operatorname{A.r}(p)=\mathbf{D}(p),
$$

where $\mathbf{r}(p)=[x(p), y(p), z(p)]^{\mathrm{T}}$ is the position vector of the moving object with unknown coordinates, $\mathbf{A}$ is the matrix of the transceivers' coordinates differences, $\mathbf{D}(p)$ is the matrix of square distances to the moving object and square modules of measurement transceivers' position vectors.

Multiply both sides of (16) with $(\mathbf{A})^{\mathrm{T}}$, i.e.,

$$
(\mathbf{A})^{\mathrm{T}} \mathbf{A} \cdot \mathbf{r}(p)=(\mathbf{A})^{\mathrm{T}} \cdot \mathbf{D}(p) \text {. }
$$

The vector-coordinates of a moving object can be computed by the expression

$$
\mathbf{r}(p)=(\mathbf{A})^{+} \cdot \mathbf{D}(p),
$$

where $(\mathbf{A})^{+}$is the pseudo inverse matrix of the matrix $\mathbf{A}$.

As the columns of the matrix $\mathbf{A}$ are independent, the pseudo inverse matrix is computed by the expression

$$
\mathbf{A}^{+}=\left(\mathbf{A}^{\mathrm{T}} \cdot \mathbf{A}\right)^{-1} \cdot(\mathbf{A})^{\mathrm{T}} .
$$

In case the measurement transceivers are stationary and positioned in the coordinate plane $O x y$, the matrix $\mathbf{A}$ becomes singular and has the form 


$$
\mathbf{A}=\left[\begin{array}{lll}
x_{21}(p) & y_{21}(p) & 0 \\
x_{31}(p) & y_{31}(p) & 0 \\
x_{4,1}(p) & y_{4,1}(p) & 0
\end{array}\right] .
$$

The zero column from the matrix $\mathbf{A}$ can be omitted, and then matrix equation can be rewritten as

$$
\left[\begin{array}{ll}
x_{21}(p) & y_{21}(p) \\
x_{31}(p) & y_{31}(p) \\
x_{4,1}(p) & y_{4,1}(p)
\end{array}\right] \cdot\left[\begin{array}{l}
x(p) \\
y(p)
\end{array}\right]=\frac{1}{2}\left[\begin{array}{l}
\hat{R}_{1}^{2}(p)-\hat{R}_{2}^{2}(p)-R_{1}^{2}(p)+R_{2}^{2}(p) \\
\hat{R}_{1}^{2}(p)-\hat{R}_{3}^{2}(p)-R_{1}^{2}(p)+R_{3}^{2}(p) \\
\hat{R}_{1}^{2}(p)-\hat{R}_{4}^{2}(p)-R_{1}^{2}(p)+R_{4}^{2}(p)
\end{array}\right] .
$$

It is worth noting that the matrix Equation (21) provides solution for the coordinates $x(p)$ and $y(p)$ of the moving point. Given $x(p)$ and $y(p)$, the coordinate $z(p)$ is calculated from one of the square Equations (3) or (4). In case Equation (3) is used both solutions for the third coordinate $z(p)$ is given by equation

$$
z_{1,2}(p)=z_{i}(p) \pm\left[R_{i}^{2}(p)-\left(x(p)-x_{i}(p)\right)^{2}-\left(y(p)-y_{i}(p)\right)^{2}\right]^{\frac{1}{2}},
$$

where the index $i$ may accept any value in the interval from 1 up to 4 .

The sign \pm is chosen in accordance with the position of the moving object in the coordinate system of observation and orientation of the plane where stationary transceivers are located. In practice, the coordinate system of observation and position of the moving object are defined in a first (positive) quadrant of the coordinate space. Then the sign is chosen "+".

\section{Experimental scenario and stages of data packet processing}

The experimental set-up of the Hexamite ultrasonic positioning system is presented on Fig. 1. The system is composed of five ultrasonic HX7TR devises designed for indoors or clear-weather outdoor operations.

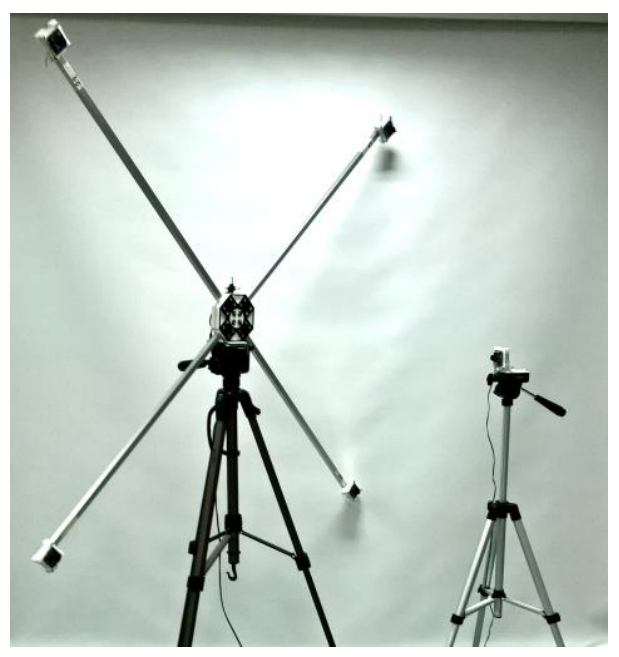

Fig. 1. Hexamite ultrasonic positioning system architecture 
The HX7TR12-IP42 broad beam transceiver, times the flight of a longitudinal (ultrasonic) wave from itself to other similar devices within range. Time of flight, determines distance to the nearest object as well as distance to targeted points. The HX7 transceiver is capable of serving as ultrasonic transmitter and/or ultrasonic receiver. Power spatial distribution as a beam pattern of the ultrasonic transceiver is depicted in Fig. 2.

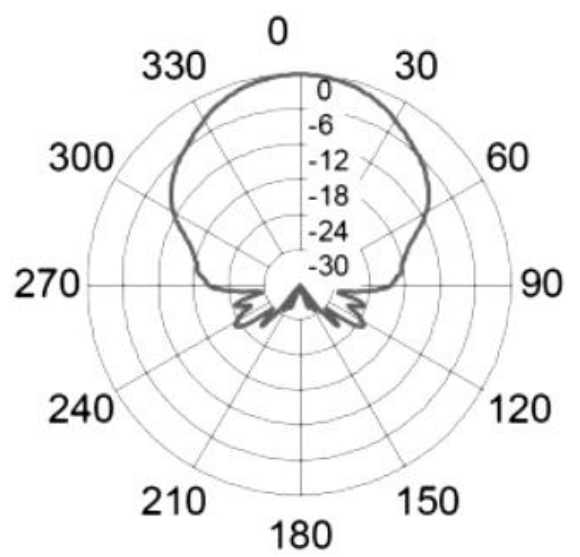

Fig. 2. HX7TR beam pattern

The maximal distance, $16 \mathrm{~m}$ covered by HX7TR is measured at $0^{\circ}$ and normalized maximal power of $0 \mathrm{~dB}$. In the spatial angle of $120^{\circ}$ the distance is limited to $10.7 \mathrm{~m}$. A Monitor/Synchronizer HX7MS that defines the order of measurements and transfer of data to the processing center performs synchronization of the functionality of all ultrasonic devices and data processing.

Hexamite HX7 Specification (http://www.hexamite.com/hx7trSpec.html) is used as a hardware measurement platform. When the system is powered, the transceivers start emitting and receiving ultrasonic pulses with $40 \mathrm{kHz}$ carrier frequency and $5 \mathrm{~ms}$ pulse duration. This way the hardware platform forms data packets and sends them by $2.5 \mathrm{GHz}$ RF to Processing Center (PC). Each data packet consists of 40 measurements ( 4 mutual connections - edges $\times 2$ measurements $\times 5$ ultrasonic devises - vertexes) and forms an oriented and weighted graph. Each vertex of the graph has 4 incident edges. The length of the edge is formed from two measurements forward and backward between two neighbor vertexes. When first data packet is fully formed, (all 40 measurements are received on the PC) then the first processing step is to determine which points are stationary and which of them is a moving point.

If two incident edges of a particular vertex have lengths equal or differ by plus/minus $10 \mathrm{~mm}$, then this vertex is accepted as a stationary point's candidate. Applying this approach to the initial data packet no more than four stationary points' candidates are determined. The data packet from 40 measurements is formed in order of arrival of each ultrasound measurement. 


\section{Measurement basis's determination and coordinates' calculation of a moving object}

The four stationary points' candidates form a square of a base pseudo pyramidal. To represent these 4 points in 3D space it is assumed that their common $z$ coordinate is zero. Ranges between each couple of vertices are averaged to form single precise distances. Then, in order to find their $x, y$ coordinates the following data block is processed (Table 1).

Table 1. Part of the data block containing the basis points' distances

\begin{tabular}{|c|c|c|}
\hline Rom, point & To, point & Distance, $\mathrm{mm}$ \\
\hline 23 & 22 & 972 \\
\hline 25 & 22 & 969 \\
\hline 24 & 25 & 980 \\
\hline 24 & 23 & 975 \\
\hline
\end{tabular}

First measurement in data block of stationary points is from 23 (point $B_{2}$ ) to 22 (point $B_{1}$ ) with length of $972 \mathrm{~mm}$. A detached coordinate system is formed and point 22 (point $B_{1}$ ) is put on a position with coordinates $x=0, y=0, z=0$, the origin of the coordinate system $(x, y, 0)$, and point 23 is put on a position with coordinates $x=972, y=0, z=0$ (on $O x$ axis). The second measurement in data block is from 25 to 22 with length of $969 \mathrm{~mm}$. Because in previous step the point 22 is already positioned with coordinates $(0,0,0)$, point 23 is positioned on $O x$ axis, then point 25 (point $B_{4}$ ) is put on a position $x=0, y=969, z=0$ (on $O y$ axis). The third measurement with length of $980 \mathrm{~mm}$ and forth measurement with length of $975 \mathrm{~mm}$ in data block determine the position of the last point 24 (point $B_{3}$ ) with coordinates $x=980, y=975, z=0$. This is illustrated in Fig. 3 .

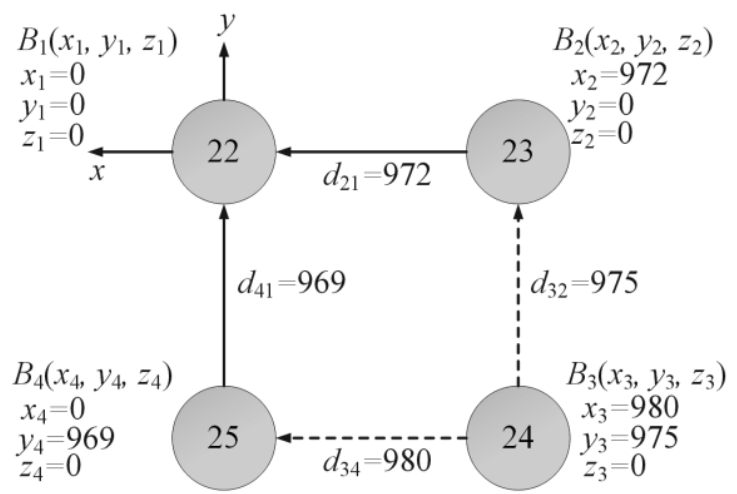

Fig. 3. Determination of pyramid base points coordinates

Based on the notations in Fig. 3 the matrix equation (15) can be expressed as

$$
\left[\begin{array}{ccc}
d_{21}(p) & 0 & 0 \\
x_{3}(p) & y_{3}(p) & 0 \\
d_{4,1}(p) & y_{4}(p) & 0
\end{array}\right] \cdot\left[\begin{array}{c}
x(p) \\
y(p) \\
z(p)
\end{array}\right]=\frac{1}{2}\left[\begin{array}{c}
\hat{R}_{1}^{2}(p)-\hat{R}_{2}^{2}(p)+d_{21}^{2}(p) \\
\hat{R}_{1}^{2}(p)-\hat{R}_{3}^{2}(p)+x_{3}^{2}(p)+y_{3}^{2}(p) \\
\hat{R}_{1}^{2}(p)-\hat{R}_{4}^{2}(p)+d_{41}^{2}(p)
\end{array}\right] .
$$


Finally, the first four points (32 measurements) that are already positioned are removed from the original data packet (40 measurements). The rest of eight measurements represent four distances measured twice between stationary points (stationary vertexes of the graph) and the 5th moving point (moving vertex of the graph). Ranges between each couple of vertices are averaged to form single precise distances. It allows minimizing errors of measurements.

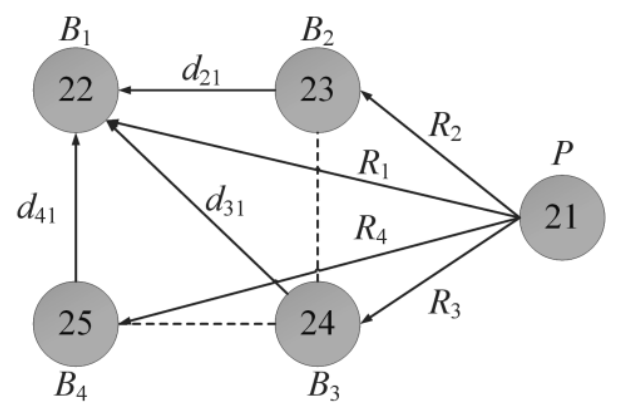

Fig. 4. Coordinate determination of unknown point

As soon as the $p$-th data packet arrives, distances measured between each couple of vertexes are averaged. Averaged distance measurements of the pseudo pyramid's basis, and averaged range differences between four points of the pseudo pyramid's basis and a moving point are considered constant in the moment of measurement; they are used to constitute the matrix Equation (15), which solution defines coordinates of unknown moving point. This assumption is applicable in two scenarios. The object with unknown coordinates is displaced from one position to another position, and for each stationary position, measurements are performed. The maximal achievable velocity of the object, (e.g., robot) is less than $0.2 \mathrm{~m} / \mathrm{s}$ and ultrasonic $\mathrm{HX} 7$ measurement pulse duration is $5 \mathrm{~ms}$, then the displacement at the moment of measurement is less than $1 \mathrm{~mm}$.

Substitute the data from Fig. 3 and Fig. 4 in (23). The following matrix equation can be written:

$$
\left[\begin{array}{ccc}
972 & 0 & 0 \\
980 & 975 & 0 \\
969 & 969 & 0
\end{array}\right] \cdot\left[\begin{array}{c}
x(p) \\
y(p) \\
z(p)
\end{array}\right]=\frac{1}{2}\left[\begin{array}{c}
\hat{R}_{1}^{2}(p)-\hat{R}_{2}^{2}(p)+(972)^{2} \\
\hat{R}_{1}^{2}(p)-\hat{R}_{3}^{2}(p)+(980)^{2}+(975)^{2} \\
\hat{R}_{1}^{2}(p)-\hat{R}_{4}^{2}(p)+(969)^{2}
\end{array}\right] .
$$

As the elements of the third column in the matrix of coefficients are zeros, the matrix equation is reduced to the form

$$
\left[\begin{array}{cc}
972 & 0 \\
980 & 975 \\
969 & 969
\end{array}\right] \cdot\left[\begin{array}{l}
x(p) \\
y(p)
\end{array}\right]=\frac{1}{2}\left[\begin{array}{c}
\hat{R}_{1}^{2}(p)-\hat{R}_{2}^{2}(p)+(972)^{2} \\
\hat{R}_{1}^{2}(p)-\hat{R}_{3}^{2}(p)+(980)^{2}+(975)^{2} \\
\hat{R}_{1}^{2}(p)-\hat{R}_{4}^{2}(p)+(969)^{2}
\end{array}\right],
$$

where $\hat{R}_{1}^{2}(p), \hat{R}_{2}^{2}(p), \hat{R}_{3}^{2}(p)$ and $\hat{R}_{4}^{2}(p)$ are distances measured by transceivers in the $p$ th moment. 
As follow from the matrix equation (25) the solution of (18) is only for unknown coordinates $x(p)$ and $y(p)$. The solution of the third coordinate $z(p)$ for $p$-th data packet is calculated by (21).

\section{Square-based pyramid visualization and program implementation}

\subsection{Square-based pyramid visualization}

Initial state of the ultrasonic system HX7 devices, visualized as an oriented and weighted graph with edges marked by current measured distances and vertexes is presented in Fig. 5a. The ultrasonic devices' numbers are shown next to the vertex and the ranges between devices in millimeters is written on the edges. State after displacement of the moving device visualized by an oriented and weighted graph with edges and vertexes is illustrated in Fig. 5b. As can be seen, there is displacement in the position of the point 21 in respect to the square pyramid basis. It means that this point is a moving object in the space, registered in time by calculation of particular number of data packets.

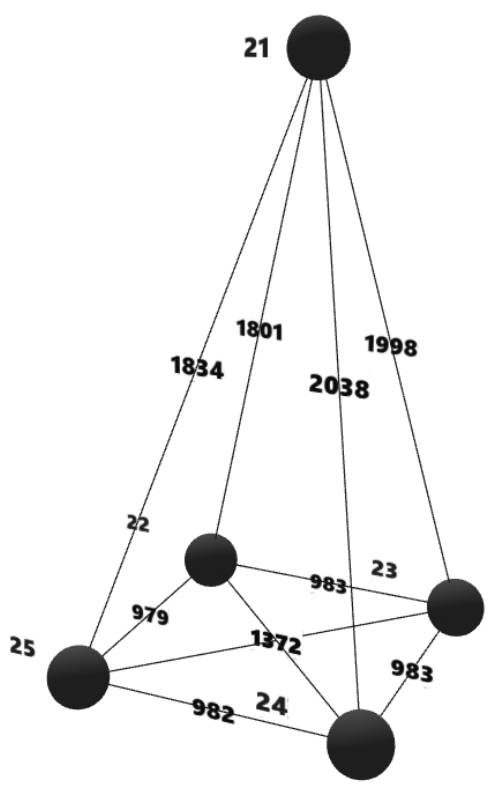

(a)

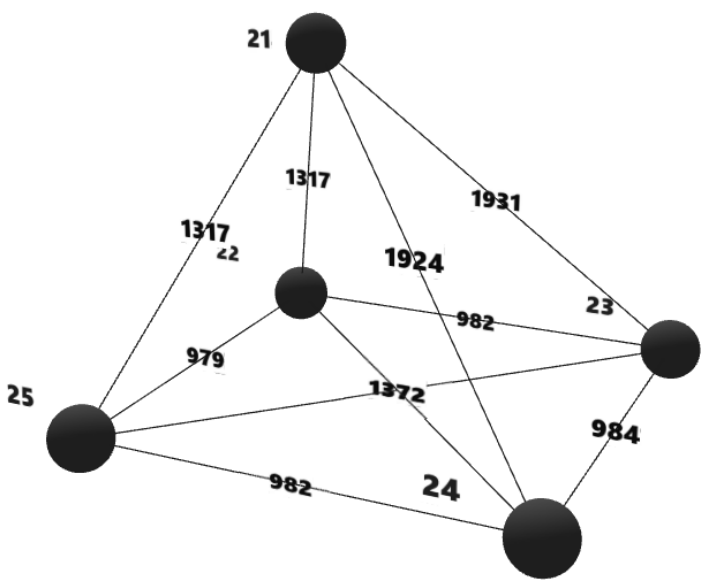

(b)

Fig. 5. 3D dynamic graphs visualizing of ultrasonic system HX7: initial state of the devices (a); state after displacement of the moving device (b)

\subsection{Program implementation}

The fragment of the source code of the application called HX7 Render is presented in Fig. 6. It is developed in C\# Programming Language using Microsoft.NET Framework 4.6 and Microsoft Visual Studio Community Edition 2015 Update 3. 
Application source code is published under GNU General Public License Version 3, GitHub Repository (https://github.com/dimitarminchev/Hexamite).

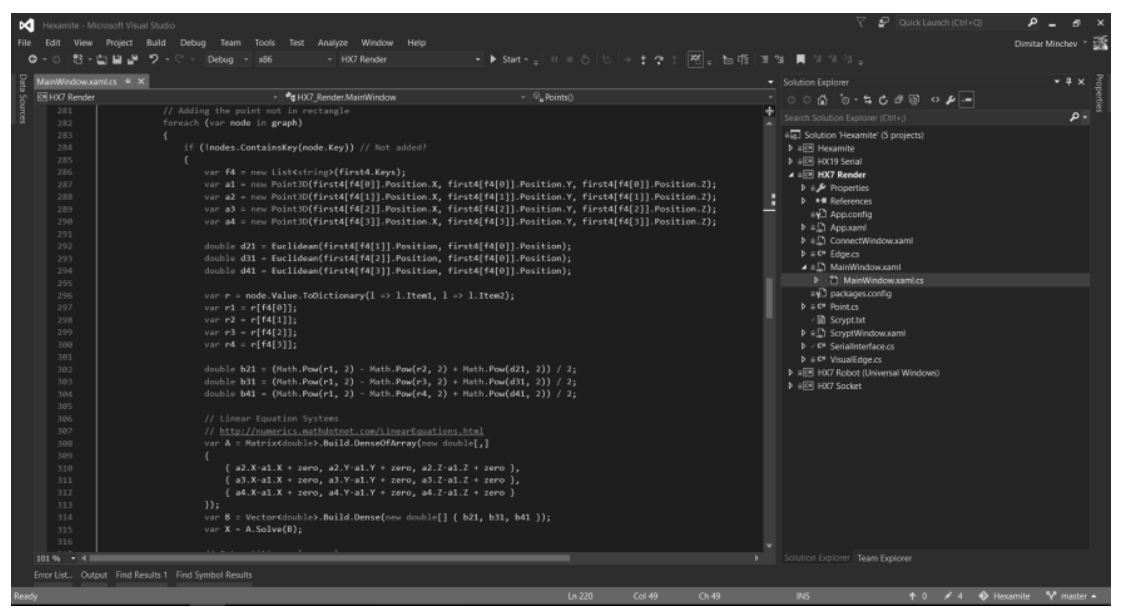

Fig. 6. Fragment of the source code

\section{Conclusion}

In the paper design and implementation of an ultrasonic positioning system based on multifunctional hardware components, newly released have been discussed. Moving object coordinates' determination has been analytically described. Matrix equations in respect of unknown coordinates of a moving object with coefficients and measurement distances has been derived. An experimental set-up has been established and described, and stages of data packet processing have been formulated. A pseudo pyramid based on measurement distances and calculated coordinates of the moving object has been built dynamically. HX7TR multifunctional ultrasonic devises, transceivers, have been used to implement the positioning system. A C\# program implementation for the coordinate determination and dynamic 3D visualization is created. The computation algorithm and its program realization as well as HX7TR ultrasonic devises can be used in development of indoor ultrasonic positioning systems embedded in different IoT systems and robotics applications.

\section{References}

1. S a h i n o g l u, Z., S. Ge z i c i, I. G u ve n c. Ultra-Wideband Positioning Systems: Theoretical Limits, Ranging Algorithms, and Protocols. New York, Cambridge University Press, 2008.

2. Jekabsons, G., V. Kairish, V. Zuravlovs. An Analysis of WiFi Based Indoor Positioning Accuracy. - Sci. J. Riga Tech. Univ. (RTU), Vol. 47, 2011, pp. 131-137.

3. A $1 \mathrm{~h} \mathrm{mi} \mathrm{e} \mathrm{d} \mathrm{at,} \mathrm{T.} \mathrm{A.,} \mathrm{S.} \mathrm{H.} \mathrm{Y} \mathrm{a} \mathrm{n} \mathrm{g.} \mathrm{A} \mathrm{ZigBee-Based} \mathrm{Mobile} \mathrm{Tracking} \mathrm{System} \mathrm{through} \mathrm{Wireless}$ Sensor Networks. - Int. J. Adv. Mechatron. Syst., Vol. 1, 2008, pp. 63-70. 
4. K a i, C., N. P i s s i n o u, K. M a k k i. Cellular Network Location Estimation via RSS-Based Data Clean Enhanced Scheme. - In: Proc. of IEEE Symposium on the Computers and Communications (ISCC'11), Miami, FL, USA, 28 July 2011, pp. 924-930.

5. J u d, D., A. M i c h e 1. Motion Tracking Systems - An Overview of Motion Tracking Methods. Spring Term, 2011. students.asl.ethz.ch/upl_pdf/308-report.pdf.

6. Pe $1 \mathrm{k}$ a, M. Position Calculation with Least Squares Based on Distance Measurements. Lübeck University of Applied Sciences: Technical Report 2015; 2. https://cosa.fh-luebeck.de/files/download/pub/TR-2-2015-least-sqaures-with-ToA.pdf.

7. Pelka, M., C. B oll me ye r, H. Hellbrü ck. Indoor Localization Based on Bi-Phase Measurements for Wireless Sensor Networks. - In: IEEE Wireless Communications and Networking Conference (WCNC'15). Track 3: Mobile and Wireless Networks (IEEE WCNC'15), New Orleans, USA, March 2015.

8. L a a r a i ed h, S., M. Y u, L. A v ri 11 o n, S. B. U gu e n, Comparison of Hybrid Localization Schemes Using RSSI, TOA, and TDOA. - In: 11th European Wireless Conference 2011 Sustainable Wireless Technologies (European Wireless), VDE, 2011, pp. 1-5.

9. Y i, S., H. Cha. An Active Tracking System Using IEEE 802.15.4-Based Ultrasonic Sensor Devices. Department of Computer Science, Yonsei University, Seodaemun-Gu, ShinchonDong 134, Seoul, Korea, pp. 120-749. https://pdfs.semanticscholar.org/6c13/5ac7fb687c3343f5f2829a082bf7760e15b0.pdf.

10. M e din a, C., J. C. S e g u r a, A. D e 1 a T orr e. Ultrasound Indoor Positioning System Based on a Low-Power Wireless Sensor Network Providing Sub-Centimeter Accuracy. - Sensors, Vol. 13, 2013, pp. 3501-3526.

11. Hashizume, H., A. Y. Sugano, K. Yat ani, M. Sugimoto. Fast and Accurate Positioning Technique Using Ultrasonic Phase Accordance Method. http://yatani.jp/paper/TenCon2005.pdf.

12. Ed u ard a s, L. V., A. A. B u 1 b e n ki e n e, P. Li n a s, A. Edgar a s. An Ultrasonic Tracking Method for Augmented Reality. http://isd.ktu.lt/it2011/material/Proceedings/5_ITA_4.pdf.

13. Gonzalez, E., L. Prados, A. J. Rubio, J. C. Segura, A. de 1 a Torre, J. M. Moya, P. Rodriguez, J. L. Mart'1n. ATLINTIDA: A Robust Indoor Ultrasound Location System: Design and Evaluation. - In: Proc. of 3rd Symposium of Ubiquitous Computing and Ambient Intelligence, Salamanca, Spain, 22-24 October 2008, Berlin-Heidelberg, Springer, Advances in Soft Computing Book Series (AINSC'09), Vol. 51, 2009, pp. 180-190.

14. Hazas, M., A. Hop per. Broadband Ultrasonic Location Systems for Improved Indoor Positioning. - IEEE Trans. Mobile Comput., Vol. 5, 2006, pp. 536-547.

15. B a e k, S. H., Y. H. K i m. Design of Multi Position Tracking System Using Ultrasonic Sensor Module. - In Proc. of Symposium on Ultrasonic Electronics, Vol. 31, 6-8 December 2010, pp. 479-480. https://www.use-jp.org/proceedings/USE10/pdf/3P-12.pdf.

16. Y ang, C.-H. A Person-Tracking Mobile Robot Using an Ultrasonic Positioning System. Department of Electrical and Computer Engineering, Naval Postgraduate School, December 2005.

17. M c C a rth y, M., H. M u 11 e r. Positioning with Independent Ultrasonic Beacons. Department of Computer Science, University of Bristol, U. K., Technical Report: CSTR-05-005. http://www.cs.bris.ac.uk/, www.cs.bris.ac.uk/Publications/Papers/2000430.pdf.

18. L a z i k, P., A. R ow e. Indoor Pseudo-Ranging of Mobile Devices Using Ultrasonic Chirps. SenSys'12, 6-9 November 2012, Toronto, ON, Canada.

19. Fil o n e n k o, V., C. C u 11 e n, J. C a r s we 11 . Investigating Ultrasonic Positioning on Mobile Phones. - In: International Conference on Indoor Positioning and Indoor Navigation (IPIN'10), September 2010, pp. 1-8.

20. S t e phe n, P. T., P. A. D ind a, R. P. Di ck, G. Me mik. Indoor Localization without Infrastructure Using the Acoustic Background Spectrum. - In: Proc. of 9th International Conference on Mobile Systems, Applications, and Services (MobiSys'11), ACM, New York, NY, USA, 2011, pp. 155-168. 
21. Arp, D., E. Quiring, C. Wressnegger, K. Rieck. A Study on Ultrasonic Device Tracking. Computer Science Report No 2016-02, Technische Universität Braunschweig, Institute of System Security. https://www.sec.cs.tu-bs.de/pubs/2016-batmobile.pdf.

22. A m u n d s o n, I., X. D. K o u t s o u k o s. A Survey on Localization for Mobile Wireless Sensor Networks. - In: Proc. of 2nd International Conference on Mobile Entity Localization and Tracking in GPS-Less Environments (MELT'09), Berlin, Heidelberg, Springer-Verlag, 2009, pp. 235-254.

23. S u n, Z., R. F a r l e y, T. K a l e a s, J. E 11 i s, K. Ch i k k a p p a. Cortina: Collaborative ContextAware Indoor Positioning Employing RSS and RTOF Techniques. - In: IEEE International Conference on Pervasive Computing and Communications Workshops (PERCOM'11 Workshops), March 2011, pp. 340-343.

24. T a rzia, S. P., P. A. D inda, R. P. D i ck, G. Me mik. Indoor Localization without Infrastructure Using the Acoustic Background Spectrum. - In: Proc. of 9th International Conference on Mobile Systems, Applications, and Services (MobiSys'11), ACM, New York, NY, USA, 2011, pp. 155-168.

25. Chivarov, N., S. S h i varo v, Ultrasonic Positioning System for Mobile Robots. - Problems of Engineering Cybernetics and Robotics, Vol. 62, 2010 pp. 43-51. 